\title{
Role of MRI in the Evaluation of Primary Bone Tumors
}

\author{
Prem Chand $\mathbf{P}^{1}$, Gurivi Reddy $\mathbf{C}^{2}$, Aswith Kumar $\mathbf{P}^{3}$ \\ ${ }^{1}$ Assistant Professor, Department of Radiology, ${ }^{2}$ Senior Resident, Department of Radiology, ${ }^{3}$ Assistant Professor, Department \\ of Radiology, Narayana Medical and College Hospital, Nellore, A.P, India.
}

Corresponding author: Dr Prem Chand P, Assistant Professor, Department of Radiology, Narayana Medical College and Hospital, Nellore, A.P, India.

DOI: http://dx.doi.org/10.21276/ijcmsr.2018.3.4.10

How to cite this article: Prem Chand P, Gurivi Reddy C, Aswith Kumar P. Role of MRI in the evaluation of primary bone tumors. International Journal of Contemporary Medicine Surgery and Radiology. 2018;3(4):D38-D43.

\section{A B S T R A C T}

Introduction: Bone tumors and tumor like conditions encompass a complex and variegated group of disorders requiring the combined efforts of the orthopedic surgeon, radiologists and the pathologists to appropriately manage. Current study was designed to assess the accuracy of M.R.I. in local staging of primary bone tumors and also to study the signal characteristics of primary bone tumors and correlate with surgical and pathological findings.

Material and Methods: A clinical suspicion of bony neoplasm (or conditions mimicking bone tumors) were first evaluated with plain radiographs taken in at least two different projections. patients with a high suspicion of primary bone neoplasms (or conditions mimicking them) and in whom surgery was viable options were referred to the radiology department for further evaluation with MRI. 68 consecutive patients were enrolled for the study and were followed up.

Results: 37 patients had primary bone malignancies out of which three were in the spine-plasmacytoma ( $\mathrm{N}=2$ ) and Chordoma $(\mathrm{N}=1) .14$ patients had metastatic disease including systemic lymphomas with secondary bone involvement. 3 patients had osteomyelitis.16 lesions were located to the axial skeleton out of which 11 were in the spine. 38 lesions were in the appendicular skeleton with the lesions around the knee joint (distal femora and proximal tibia) being the commonest. Osteosarcoma was the most common primary malignant bone tumor and Giant cell tumor was the most common benign bone tumor.

Conclusion: MRI is a very accurate tool for staging primary bone malignancies and should be obtained whenever possible in a suspected case of primary bone malignancy for accurate Enneking staging. STIR and T1 weighted sequences are equally efficacious for measuring the intramedullary tumor length and either one of them can be used for the same. Though MRI does not allow a specific diagnosis and its main advantage is surgical staging in a few cases it does contribute vital new diagnostic information.

Key words: Bone Malignancy, MRI, Surgical Staging

\section{INTRODUCTION}

Bone neoplasms can be broadly classified as benign and malignant. Approximately 70\% of malignant neoplasms are metastatic and the rest are primary in nature. Multiple myeloma and osteosarcoma are the most common primary malignant neoplasms while benign bony neoplasms include osteomas, chondromas etc and cystic lesions like simple bone cyst. Then there are tumor-like processes like fibrous dysplasia which because of their varied radiographic appearance can mimic any other disease process.

Plain radiographs are usually the first line of investigation when a patient presents with a bony swelling. They remain the most valuable tool for establishing a diagnosis or differential diagnosis for bony neoplasms and determining whether the lesion is benign or aggressive. Lesions which are poorly marginated and show a wide zone of transition, moth eaten or permeative appearance and spiculated or poorly organized periosteal reaction are likely to be malignant or aggressive. Likewise well marginated lesions with a narrow zone of transition and showing a geographic pattern with continuous organized periosteal reaction are more likely to be benign. Vital information required to arrive at a differential diagnosis like age, location, pattern of destruction, periosteal involvement, matrix mineralization are all provided by plain radiographs. MDCT with its multiplanar reconstruction further refines the information obtained on plain radiographs. However both the modalities are poor at defining the soft tissue extension and local staging.

Although M.R.I. can be used to narrow the differential diagnosis, it is unwise to attempt making a specific diagnosis on M.R.I. without conventional radiography. ${ }^{1}$ The capability ofMRI to distinguish benign from malignant musculoskeletal lesions remains controversial. ${ }^{2}$ A careful study by Crim and colleagues warned that the ability to distinguish benign from malignant soft-tissue masses with MRI was only $50 \%$ for benign masses and $80 \%$ for malignant masses. ${ }^{3}$

CT and plain radiographs were both inadequate in this regard. The impact of M.R.I. in local staging was unparalleled in that its exquisite soft tissue delineation and multiplanar capabilities allowed a confident diagnosis regarding muscle, 
neurovascular, articular and joint involvement. It was also superior in assessing marrow involvement and skip metastasis and hence has become indispensible in the preoperative evaluation of bone tumors. Of late M.R.I is also increasingly being used to determine the response of the primary bone tumor to neo adjuvant chemotherapy. M.R.I. has not been shown to be significantly better than plain radiographs in predicting the nature of the lesion, that is, as benign or aggressive and in arriving at a differential diagnosis. However few bony lesions do show typical signal characteristics on MRI (like the very bright signal of chondroid tumors on T2 weighted images, fluid-fluid levels etc). This study aimed to evaluate the role M.R.I. in evaluating a patient who presents with a bony swelling.

\section{MATERIAL AND METHODS}

Study was done in the Department of Radiology, Narayana medical college hospital, Nellore, A.P, India for the period of two years after taking the informed consent from the subjects and ethical clearence from the etical board.

All patients presenting to the orthopedics department with a clinical suspicion of bony neoplasm (or conditions mimicking bone tumors) were first evaluated with plain radiographs taken in at least two different projections. After clinocoradiological analysis of the plain radiographs, patients with a high suspicion of primary bone neoplasms (or conditions mimicking them) and in whom surgery was viable options were referred to the radiology department for further evaluation with MRI. In view of this referral pattern this study contains a disproportionate number of primary bone tumors compared to metastasis. 68 consecutive patients were enrolled for the study and were followed up.

\section{Exclusion criterion}

Patients lacking a histopathological diagnosis. 14 patients were excluded

\section{MRI Technique}

MRI was performed with a 3 TESLA MR imaging system, GE Healthcare, USA.

Patient was positioned head first and supine into the gantry. Dedicated coils were used for the part to be imaged-shoulder, elbow and knee coils. Body coils were used for imaging the pelvis and chest.

Initially a T1 weighted gradient echo localizer was obtained in three orthogonal planes.T1 SE (spin echo) images was obtained in the region of interest in the coronal or sagittal plane to delineate the lesion. This was followed by sagittal or coronal STIR sequences and axial SE T1 and SE T2 weighted images. T2 gradient echo images in the axial plane were taken if hemorrhage was suspected. A large field of view T1 or STIR image including the entire bone in the coronal or the sagittal plane was also taken to identify any skip lesions. Post contrast T1 weighted images were obtained in all the three planes. In a few patients post contrast images could not be taken due to economic constraints $(\mathrm{N}=20)$

\section{Image analysis}

The MR images were evaluated on the following lines.

Location: The bone involved and the location along the long axis of the bone.

Signal characteristics: The signal in the tumor was graded as dark or bright relative to the adjacent muscle on the various pulse sequences.

Margins: Margins of the lesion were classified as well defined or ill defined based on the transition between the tumor signal to the normal marrow signal.

Edema: Ill defined diffuse STIR signal surrounding the bony lesion and ill defined STIR bright signal with a feather like appearance following the facial planes without focal mass effect was identified as edema ${ }^{75}$. Edema was tabulated as present $(\mathrm{Y})$ or absent $(\mathrm{N})$.

Contrast enhancement: Contrast enhancement was graded as homogeneous, heterogeneous or septal like.

Intramedullary tumor length: The intramedullary length of the tumor was measured in long axis in centimeters -either coronal or sagittal images on T1 weighted, STIR and post contrast fat saturated T1 weighted images.

Skip lesions: Skip lesions assessed in T1/STIR weighted sequences in the long axis of the bone-either coronal or sagittal images. Presence(Y) or absence $(\mathrm{N})$ of cortical involvement and destruction. Presence( $\mathrm{Y}$ ) or absence $(\mathrm{N})$ of soft tissue component. Joint involvement: Joint involvement was tabulated as present $(\mathrm{Y})$ or absent $(\mathrm{N})$. Joint involvement was presumed $(\mathrm{Y})$ when the tumor was extending into the joint space either by destruction of the articular cartilage or joint capsule. Enhancement of intracapsular structures like the cruciate ligaments, tumor abutting the articular cartilage with no clear fat plane between the tumor and articular cartilage were called probable joint involvement. For the purpose of statistical analysis probable joint involvement was taken as presence joint involvement(Y) on MRI.

Neurovascular involvement: Neurovascular invasion was tabulated as present $(\mathrm{Y})$ or absent $(\mathrm{N})$. Neurovascular involvement was presumed when the tumor was completely surrounding these structures or containing at least half the circumference with loss of the adjacent fat plane. "Probable" was used when the tumor was just abutting the neurovascular bundle (less than half the circumference). For the purpose of statistical analysis probable neurovascular involvement was taken as presence $(\mathrm{Y})$ of neurovascular involvement.

The MRI findings were then correlated with the surgical and histopathological (HPE) findings which were taken as gold standard.

To test the accuracy of MRI in local staging the following parameters were evaluated namely intramedullary tumor length, joint invasion, neurovascular invasion, cortical breaks and soft tissue component.

The intramedullary tumor length as measured on MR images in centimeters (in T1, STIR and post contrast T1) was correlated with the measurements taken on the HPE resection specimen using Spearman's rho correlation analysis. Wilcoxsons rank test was performed (as the data was non parametric) to test significance of difference between HPE Vs T1 weighted and HPE Vs STIR weighted measurements and the $\mathrm{p}$ value was obtained. 


\section{RESULTS}

54 patients were studied during a period of two years. 37 patients had primary bone malignancies out of which three were in the spine-plasmacytoma $(\mathrm{N}=2)$ and Chordoma $(\mathrm{N}=1) .14$ patients had metastatic disease including systemic lymphomas with secondary bone involvement. 3 patients had osteomyelitis (figure-1).

16 lesions were located to the axial skeleton out of which 11 were in the spine. 38 lesions were in the appendicular skeleton with the lesions around the knee joint (distal femora and proximal tibia) being the commonest.

Osteosarcoma was the most common primary malignant bone tumor and Giant cell tumor was the most common benign bone tumor. Most of the metastatic lesions were in the spine and systemic lymphoma with bone involvement was the most common metastatic disease followed by carcinoma lung.

This does not reflect the normal distribution of bony

\begin{tabular}{|l|c|c|}
\hline Diagnosis & Number & $\begin{array}{c}\text { Mean age } \\
\text { in years }\end{array}$ \\
\hline Osteosarcoma & 14 & 20 \\
\hline Chondrosarcoma & 6 & 48.3 \\
\hline $\begin{array}{l}\text { Metatasis including secondary } \\
\text { lymphomas }\end{array}$ & 14 & 59.9 \\
\hline Giant cell tumor & 7 & 35 \\
\hline Plasmacytoma & 2 & 37 \\
\hline Simple bone cyst & 2 & 45 \\
\hline Osteomyelitis & 3 & 16 \\
\hline Ewings sarcoma & 1 & 9 \\
\hline Anursymal bone cyst & 1 & 11 \\
\hline Chondroblastoma & 1 & 14 \\
\hline Hemanigioma Table-1: Age distribution \\
\hline Enchondroma & 1 & 60 \\
\hline Chordoma & 1 & 28 \\
\hline \multicolumn{2}{|l}{} \\
\hline
\end{tabular}

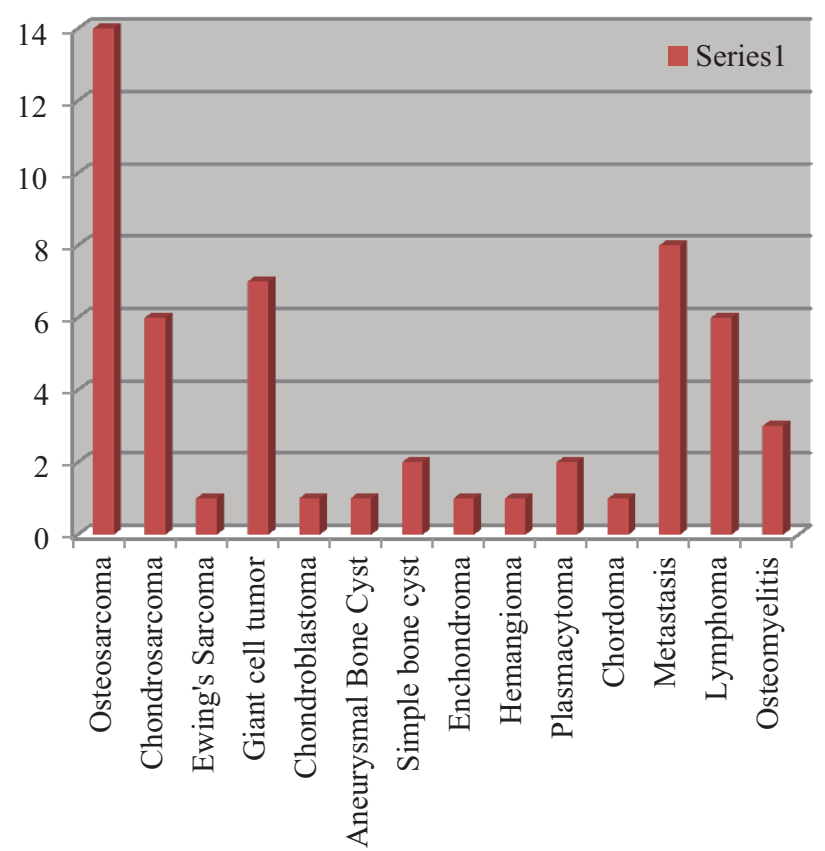

Figure-1: Frequencies of bone tumors. neoplasms-as metastasis is the most common bony neoplasm. This is because our cases were referred for MRI after the clinocoradiological evaluation of the plain radiographs and only those cases thought to represent primary bone malignancies amenable to surgery were further evaluated with MRI. As a result metastasis is grossly underrepresented and so also benign tumors like osteoid osteoma since their plain radiographic appearances are typical enough to make a diagnosis and MRI will not be useful diagnostically or therapeutically.

Most common malignant primary bone tumor: Osteosarcoma=14.

Most common benign primary bone tumor: Giant cell tumor's $=6$.

Most common metastatic lesion in bone: Secondary bone involvement due to systemic Non Hodgkin's lymphoma $(\mathrm{N}=6)$ followed by carcinoma lung (figure-1).

Out of the 37 cases of primary bone malignancies 26 underwent resection, 4 cases (two simple bone cysts, two giant cell tumors) underwent intralesionsal curettage and bone grafting, 1 case was not operated in view of advanced nature of the disease(osteosarcoma pubis; Patient No 15), 4 cases were lost to follow up after the histopathological diagnosis by biopsy(Ewing's sarcoma, Hemangioma, Grade 1 chondrosarcoma, Chordoma) and the two primary lesions in the spine received radiotherapy after the diagnosis (plasmacytoma) (table-1).

In one case of osteomyelitis (Patient No 34) surgery was performed first since MRI revealed multiple soft tissue abscesses with a ring enhancing lesion in the bone. Surgery revealed multiple abscesses and curetting and biopsy from the bony lesion and soft tissue confirmed the preoperative diagnosis and raised the possibility of tuberculous etiology. Biopsy was performed before surgery in the other two cases of osteomyelitis (Patient No36, 41); biopsy revealed no evidence of malignancy with features favoring osteomyelitis and operative findings were conclusive of osteomyelitis.

Hence to assess staging efficacy of MRI 26 cases (those which underwent resection) were available and 37 cases of primary bone malignances were used to study their signal characteristics.

\section{MRI in estimating tumor length}

Surgicopathological correlation was possible in 24 out of the 26 operated specimens. In two cases tumor involved the flat bones and hence intramedullary length was not calculated. TI weighted contrast images were available only in 18 out of the 24 cases while T1 weighted and STIR images were available in all the 24 cases.

Since the data was non parametric Wilcoxson's single rank test was performed for HPE-T1 Weighted and HPE-STIR measurement comparison.

The calculated $\mathrm{p}$ value $>0.05$ for both the comparisons and hence the measurements on T1 weighted and STIR images are not significantly different from the measurements taken on histopathological specimen (gold standard).

Spearman's rho correlation co efficient showed excellent correlation between measurements taken in histopathological specimens and with MRI images. Measurements taken on T1 weighted images had the best correlation with the HPE 
measurements, however STIR and post contrast T1 weighted images also yielded good results.

These results show that either STIR or T1 weighted images could be used for estimating the tumor length. Numbers in parenthesis are $95 \%$ confidence limits

The above data indicates that MRI is a very accurate test for local staging of primary bone tumors. The $p$ value was obtained by $\mathrm{McNemar}$ test and since the $\mathrm{p}>0.05$ for all the cross tabulations it can be concluded that the findings of MRI are not statistically significantly different from the histopathological findings and hence MRI performs well in staging primary bone tumors

\section{Signal characteristics}

All our cases showed dark on T1 weighted images and bright on T2 weighted images with varying degrees of heterogeneity; except two cases of plasmacytoma showed relative dark T2 signal

Focal very dark T2 and T1 signal was seen in three cases of osteosarcoma(Patients No 7,8,10) which was confirmed on histopathology as osteoid and in one case of chondrosarcoma(case not operated)

Fluid-fluid levels were seen in four cases-aneurysmal bone cyst, Chondroblastoma and two cases of osteosarcoma (one of them being giant cell variety). The fluid-fluid levels were best visualized on $\mathrm{T} 2$ weighted images.

Hemorrhage was found in one case of osteosarcoma (patient no 18) and was confirmed on histopathology.

Skip lesions were noted in three cases (Patient No 15, 17, 19)-namely, chondroblastic osteosarcoma (pubic bone), chondrosarcoma grade 1(Ilium) and dedifferentiated chondrosarcoma (femoral metadiaphysis). Patient No. 15(chondroblastic osteosarcoma) was not operated in view of very advanced nature of disease-skip lesions, lung metastasis and hydronephrosis. Patient No 17(grade 1 chondrosarcoma) was lost to follow up. Patient No 19 (dedifferentiated chondrosarcoma) underwent radical dissection but succumbed to the disease 6 months later due to chest metastasis. All the skip lesions were much better visualized with STIR sequence than with T1 weighted images.

\section{Edema}

Edema was evaluated in STIR sequence. Intraosseous edema was present in 13 cases out of the 24 malignant bone tumors and 6 cases out of 13 benign tumors. Presence of edema as a predictive factor for malignancy was analyzed using $\mathrm{X}^{2}$ test.

\section{Pattern of contrast enhancement}

Out of the 28 patients having contracted enhanced scans 17 cases showed a heterogeneous enhancement pattern (including septal type of enhancement) and 11 case showed homogeneous enhancement pattern.

\section{Independent sample $X^{2}, p=0.12$, not significant \\ Heterogeneous enhancement pattern is not a reliable indicator of malignancy.}

Enhancement pattern and signal characteristics of the chondroid tumors were evaluated. In our case series we had 6 cases of chondrosarcoma, one case each of Enchondroma and Chondroblastoma out of which contrast scans were available in 7. All the chondroid tumors except chodroblastoma showed very bright T2 signal and showed a peripheral/septal enhancement and only one non chondroid tumor (aneurysmal bone cyst) showed the septal pattern of enhancement.

Change of differential diagnosis/significant new diagnostic information after MRI(excluding lesions of the spine, $\mathrm{N}=43$ ). Out of the "Y" three cases were osteomyelitis, one chondrosarcoma and one metastasis.

All the 11 lesions in the spine were much better characterized diagnostically with MRI when compared to plain radiographs.

\section{DISCUSSION}

The diagnosis and workup of primary bone tumors requires a multimodality approach and close cooperation between the orthopedician, radiologist and the pathologist. It is not an overemphasis to state that plain radiographs remain the single most important radiological investigation for establishing a differential diagnosis and is the most reliable predictor of the histology and the aggressiveness of the lesion. Few lesions which can be confidently diagnosed on plain radiography like fibrous cortical defect require no further imaging and can be followed up serially by radiography. However if the differential diagnosis after evaluation of plain radiographs includes primary bone malignancies further imaging is required before performing biopsy to stage the disease preoperatively. The rapid advances in chemotherapy have paved way for limb saving surgeries which in turn fostered a great interest in accurate local staging of primary bone sarcomas. Of all the modalities MRI has emerged as the best modality for staging primary bone malignancies. ${ }^{1}$ Though the impact of MRI on making a specific diagnosis has been less dramatic it can certainly increase the diagnostic confidence and has also emerged as a major tool in monitoring tumor response to chemotherapy and detecting local recurrence.

Studies over the past decade have shown MRI to be definitely superior over CT when assessing the local extent of primary bone tumors, however the capability of MRI to distinguish between benign and malignant musculoskeletal lesions remains to this day controversial. MRI has been found to be a very sensitive tool in assessing joint involvement, neurovascular and soft tissue invasion. ${ }^{4}$ In our study of 37 patients with primary bone malignancies we attempted to assess the efficacy of MRI in the local staging of primary bone malignancies and also analysed factors like presence of intraosseous edema and enhancement pattern as possible predictors of aggressiveness of the lesion

Onikul et al., studied 20 patients with osteosarcoma in the long bones and concluded that STRI images significantly overestimated tumor length while no significant differences were noted between measurements made at pathological examination and those taken on $\mathrm{T} 1$ weighted images and concluded that $\mathrm{T} 1$ weighted images must be used for assessing the intramedullary tumor extent. Edema accompanying the bone tumor was regarded as responsible for tumor length overestimation on STIR images. ${ }^{5}$

Rita Golfieri et al., retrospectively analyzed $68 \mathrm{MRI}$ studies in primary bone tumors and found that STIR images to be equally good compared to the $\mathrm{T} 1$ spin echo sequence in assessing intramedullary extent. ${ }^{6}$

Our study seems to concur with the findings of Rita Golfieri et al, and we suggest that either STIR or T1 weighted images 
could be used to assess tumor length. Increased awareness regarding intraosseous edema might be the factor which resulted in good correlation between STIR images and the actual histopathological measurements.

Schima et al, studied 95 patients with osteosarcoma and concluded that $\mathrm{T} 1$ weighted images and post contrast T1 weighted images were ideal for assessing joint invasion and demonstrated a sensitivity of $100 \%$ for joint invasion using T1 weighted images; but the specificity was only $63 \%$ and hence due to a large proportion of false positives MRI significantly over staged the disease. The most common pattern for tumor involvement in the knee was spread along the cruciate ligaments. ${ }^{7}$

Van Trommel et al, studied 34 patients with osteosarcoma and found the sensitivity, specificity positive predictive value of MRI in assessing joint invasion to be $100 \%, 70 \%$ and $86.4 \% .^{8}$

In our case series out of the 26 patients only 2 patients showed joint involvement. Both the cases were Giant cell tumors involving the lower end of femur subarticular location. MRI correctly predicted joint invasion in both these cases and hence was highly sensitive with a sensitivity of $100 \%$. Both these cases involved direct extension of tumor tissue into the patellofemoral joint with involvement of the patellar articular surface. Both the cases had joint effusion with nodular deposits in the synovium. Patient No 26 also had direct extension of tumor into the medial collateral ligament which was confirmed on surgicopathological correlation. These findings were best appreciated on T1 weighted images as it was difficult to differentiate tumor/edema interface in T2/STIR images. Contrast enhanced images were available in one of them and it showed nodular enhancement of the synovial deposits.

Three other cases which were thought to represent joint involvement in MRI were found to be negative on surgicopathological correlation. In patient no 29(giant cell tumor tibia subarticular location) enhancement and STIR signal was noted along the posterior cruciate ligament with posterior cortical break. In patient no 25 (giant cell tumor distal radius) tumor was seen up to the articular surface on MRI with no plane (fatty marrow) between the tumor and articular surface and enhancement was noted along the scapholunate joint. In patient no 3(secondary osteosarcoma tibia subarticular location) there was STIR signal and enhancement deep to the medial collateral ligament. However in none of the above cases was there a direct extension of the tumor across the joint space. The sensitivity, specificity, positive predictive value, negative predictive value and accuracy of MRI for detecting joint involvement was 100\%, 87.5\%, 40\%, 100\%, 88.6\%.

Joint effusion was found in 5 out of the 26 cases out of which only 2 had joint invasion. Both the cases with joint involvement had joint effusion. Thus joint effusion may be both reactive and related to tumor involvement leading to low positive predictive values and conversely absence of joint effusion has a high negative predictive value for joint invasion.

We found the tumor extension into the patellofemoral joint and the suprapatellar recess was the most common pattern of spread. Our findings suggest that joint involvement in
MRI can be predicted when there is a direct breach of the articular surface or capsular structures by the tumor with extension into the joint space. STIR signal and enhancement of the intracapsular structures in the absence of direct extension of the tumor into the joint space is not conclusive of joint involvement and probably represent edema. The bright signal of edema may appear similar to the tumor on STIR/T2 weighted images and hence T1 weighted images supplemented with contrast images should be used to assess joint invasion.

In our case series of 26, none of the cases showed neurovascular involvement on surgicopathological correlation; however probability of neurovascular involvement was suggested in one case on MRI (pateint No 29-giant cell tumor tibia) since tumor was in contact with the vessel for less than 180 degree of arc with no clear fat and for statistical analysis this was taken as presence of neurovascular involvement on MRI. At surgery the surgeon was able to get a clear plane between the tumor and the vessels and there were no features to suggest neurovascular involvement. As there were no true positives in our sample the sensitivity and positive predictive value were 0 . The specificity, negative predictive value and accuracy were $96.1 \%, 100 \%$ and $96.1 \%$ respectively. On the basis of our findings we found that loss of fat plane between tumor and the vessel for less than 180 degrees of arc does not constitute neurovascular invasion. However in view of lack of true positive cases we could not arrive at the criterion in MRI which could actually represent neurovascular involvement.

Cortical break and soft tissue component are important to detect as it makes the disease extracompartmental and upstages the Enneking staging. In our study 20 out of the 26 operated cases showed evidence of cortical break while MRI showed cortical break in 22 cases. MRI accurately identified all the true positive cases (20); however MRI had 2 false positive cases. In both these cases (both cases of osteosarcoma, patients 7,11 ) cortex was grossly thinned and ballooned out with a large subperiosteal soft tissue simulating a cortical break with soft tissue. The same results were obtained for soft tissue component-MRI had 2 false positive cases-the cases with large subperiosteal soft tissue (patients 7,11) were predicted as having soft tissue component. Cortical break and soft tissue component were both best seen T2/STIR images as the the bright signal of tumor stood out in contrast to the dark signal of cortical bone and muscle. The sensitivity, specificity, positive predictive value, negative predictive value, accuracy of MRI for detecting cortical break and soft tissue component are 100\%,66.7\%,90.9\%,100\% and $92.3 \%$.

Thus for surgical staging of primary bone malignancies MRI is an excellent modality with high accuracy and very good correlation with surgicopathological findings.

The specificity, sensitivity, positive predictive value, negative predictive value and accuracy of plain radiographs for detecting cortical break and soft tissue component were significantly lower when compared to MRI especially the ability of plain radiographs in picking soft tissue component. Most of the tumors were T2 bright/T1 dark with varying degree of heterogeneity and hence signal characteristics were not useful for predicting the aggressiveness of the lesion Both intraosseous and soft tissue edema accompanying musculoskeletal neoplasms have been reported earlier. 
Kroon et al, reviewed 66 cases with primary bone tumors and concluded that intraosseous edema is a frequent MRI finding in both benign and malignant neoplasms and the mere presence of edema is an unreliable indicator of the biological potential of the lesion. In the same study Kroon et al reported that the extent of edema was more extensive in benign lesions. ${ }^{9}$

De Beuckeleer HL et al, in a study of 79 cartilaginous tumors reported a high specificity(90\%) for ring and arc/septal enhancement and high signal intensity on T2 weighted images for differentiating chondroid from non chondroid tumors. ${ }^{10}$

The list of possible differential diagnoses was arrived at after the clinocoradiological evaluation of plain radiographs. However in a few cases significant new findings in MRI were encountered which either lead to narrowing/changing of the differential diagnosis. The number of cases in which MRI had an impact on diagnosis are 5; and in all the cases involving the spine $(\mathrm{N}=11)$ MRI characterized the lesion better than plain radiographs diagnostically.

In both these cases the differential diagnosis entertained after evaluation of plain radiographs were eosinophilic granuloma and primary bone malignancy apart from osteomyelitis. Both these cases underwent surgical decompression which revealed pus filled cavities in the bone and soft tissue abscess in patient 34 .

In patient no 41 MRI showed diffuse STIR signal, extensive soft tissue edema and no obvious mass lesion. Introperatively pus was seen in the medullary cavity with granulation tissue and the histopathology was negative for malignancy.

In patient no 21 the plain radiographs revealed destruction of the anterior end of the $7^{\text {th }}$ rib with a soft tissue density lesion and hence the differentials were metastasis (older age group)/pleural based tumor or peripheral lung mass invading the lung/Askin tumor/primary bone malignancy like chondrosarcoma or plasmacytoma. No matrix calcification was evident on plain radiographs. MRI revealed the lesion to be entirely arising from the anterior end of the $7^{\text {th }}$ rib with a dumbbell shaped soft tissue component, very bright T2 signal and a characteristic septal enhancement. These findings together with the typical ring and arc calcification which were seen on CT scan allowed the specific diagnosis of chondrosarcoma to be made. On histopathology lesion was a grade $2 / 3$ chondrosarcoma.

In patient no 30 an aggressive lesion was noted on plain radiographs in the subarticular region of the humerus and the differentials considered were metastasis (in view of old age)/ chondrosarcoma. MRI revealed in addition to the lesion very bulky and large axillary and supraclavicular lymph nodesmaking metastasis/lymphoma more likely. Patient also had a CT chest and abdomen which showed multiple lymph nodes and histopathology revealed non Hodgkin's lymphoma with secondary bone involvement.

All the 11 lesions in the spine were better characterized on MRI than on plain radiographs-this is because of the superior ability of MRI in detecting pre and par vertebral soft tissue and disc involvement which are crucial findings in formulating a differential diagnosis. Two lesions involving the sacrum (metastasis) and one lesion involving the clivus (Chordoma) were not visualized on plain radiographs.
Thus though MRI is not specific in making a diagnosis in a few cases it can contribute vital new information to the diagnostic information available.

\section{CONCLUSION}

MRI is a very accurate tool for staging primary bone malignancies and should be obtained whenever possible in a suspected case of primary bone malignancy for accurate Enneking staging. STIR and T1 weighted sequences are equally efficacious for measuring the intramedullary tumor length and either one of them can be used for the same.

\section{REFERENCES}

1. Baweja S, Arora R, Singh S, Sharma A, Narang P, Ghuman S, Kapoor SK, Puri S. Evaluation of bone tumors with magnetic resonance imaging and correlation with surgical and gross pathological findings. Indian Journal of Radiology and Imaging. 2006;16(4):611.

2. Pang KK, Hughes T. MR imaging of the musculoskeletal soft tissue mass: is heterogeneity a sign of malignancy?. Journal-Chinese Medical Association. 2003;66(11):655-61.

3. Crim JR, Segger LL, Yoo L, Chancnani V, Eckardt JJ. Diagnosis of soft tissue masses with MR imaging: can benign masses be differentiated from malignant ones? Radiology 1992;185(3): 581-6

4. Lietman SA, Joyce MJ. Bone sarcomas: Overview of management, with a focus on surgical treatment considerations. Cleve Clin J Med. 2010;77(Suppl 1):S8-12.

5. Ella Onikul, Barry D Fletcher Accuracy of MR imaging for estimating intraosseous extent of osteosarcoma AJR November 1996;167(6):1211-1215.

6. R Golfieri, H Baddeley. The role of STIR sequence in magnetic resonance imaging examination of bone tumors BJR 1990;63(5):251-256.

7. Schima W, Amann G, Stiglbauer R, Windhager R, Kramer J, Nicolakis M, Farres MT, Imhof H. Preoperative staging of osteosarcoma: efficacy of MR imaging in detecting joint involvement. AJR. American journal of roentgenology. 1994;163(5):1171-5.

8. Van Trommel MF, Kroon HM, Bloem JL, Hogendoorn $\mathrm{PC}$, Taminiau AH. MR imaging based strategies in limb salvage surgery for osteosarcoma of the distal femur. Skeletal radiology. 1997;26(11):636-41.

9. Kroon H.M., Bloem J.L., Holscher H.C., van der Woode H.J., Reijnierse M., Taminiau A.H.M. MR imaging of edema accompanying benign and malignant bone tumors. Skeletal Radiol. 1994;23(2):261-269.

10. De Beuckeleer LH, De Schepper AM, Ramon F. Magnetic resonance imaging of cartilaginous tumors: is it useful or necessary?. Skeletal radiology. 1996;25(2):137-41.

\section{Source of Support: Nil; Conflict of Interest: None}

Submitted: 20-09-2018; Accepted: 20-10-2018; Published online: 13-11-2018 